\title{
Estrous Behavior Suppression in a Thoroughbred
}

\section{Mare}

\section{Sang Kyu Lee \\ Inhyung Lee Le* $^{2, *}$ \\ ${ }^{1}$ KRA Veterinary Center, Korea Racing Authority, Gwacheon 13822, Korea \\ ${ }^{2}$ Department of Veterinary Clinical Scienc- es, College of Veterinary Medicine and Research Institute for Veterinary Science, Seoul National University, Seoul 08826, Korea}

*Correspondence: inhyunglee@snu.ac.kr

\section{ORCID}

Sang Kyu Lee:

https://orcid.org/0000-0002-8117-914X

Inhyung Lee:

https://orcid.org/0000-0002-0712-7165

Copyright $\odot$ The Korean Society of Veterinary Clinics
Abstract A 4-year-old Thoroughbred mare was brought to the Korea Racing Authority Equine Hospital with heightened sensitivity to touch of the hindquarter, reluctance to step forward, frequent urination even during training, clitoral winking, tail swinging, tail lifting, and training difficulties due to being in constant heat. The trainer claimed that she was in heat and exhibited reduced performance. Physical and lameness examinations revealed no abnormality. Rectal palpation and transrectal ultrasonography revealed normal reproductive organs and estrus. As she exhibited typical estrous behavior without any other physical conditions, the mare was considered to express undesirable estrous behavior. An intrauterine glass ball was inserted into the mare on ovulation day. However, the estrous behaviors were reduced unsatisfactorily. Additionally, $1 \mathrm{~mL}$ of peanut oil was administered on the 10 th day after the intrauterine device insertion. The mare returned to training and expressed no undesirable estrous behavior over 30 days after the peanut oil treatment. However, further follow-ups were not conduct as the mare was retired to a farm after a limb injury occurred during training. This is the first report of undesirable estrous behavior in a Thoroughbred mare under the rules of racing in Korea. It is recommended that equine clinicians in Korea consider peanut oil as a therapeutic agent to control mares' estrous behavior-related problems.

Key words estrous behavior, estrus suppression, peanut oil, thoroughbred, mare. 


\section{Introduction}

Horses are seasonally polyestrous animals during the breeding season with an estrous cycle of 21 days (15). Mostly, estrus is expressed for 5-7 days in each cycle with the expression of estrous behavior affected by two hormones: estrogen and progesterone (15). Horses display the greatest variation in estrous cycle length and estrous behavior under the influence of high estrogen and low progesterone levels $(2,12)$. The strength of estrous behavior in the intact mare varies, exhibiting very strong and/or exaggerated symptoms (4). In addition, the estrous behavior of mares can be expressed for varying periods with different intensities from one mare to another (18). A mare's typical estrous behaviors include tail lifting, clitoral winking, breeding posture, full urination, and resistance toward moving forward $(13,18)$. Such excessive estrous behavior can cause difficulties in the training and handling of athletic mares (4). These changes in temperament and tractability occur in association with estrus and can degrade performance (13). According to a survey by Jorgensen et al. (9) in 1996, 671 out of 751 equine practitioners deemed estrous behavior to influence a mare's athletic performance. Trainers and owners commonly request veterinarians to suppress estrous signs, resulting in reduced performance in athletic mares (15). The administration of progesterone has been the most commonly used treatment method with good successful outcomes for the suppression of estrus and to treat excessive estrous behavior in mares (18). Altrenogest is a synthetic progesterone approved for use in horses to suppress estrus by daily oral administration (11). It is considered the gold standard for suppressing estrous behavior in mares (21). However, the administration of any drugs that affect race performance is strictly regulated by the rules of horse racing in some countries, including Korea $(1,6,7,10,16)$.

Considering the fair number of athletic horses in Korea, there are mares exhibiting estrous behavior problems in Korea. This study provides is the first case of equine estrous behavior suppression under the rules of racing in Korea.

\section{Case Report}

A 4-year-old Thoroughbred mare was presented to the Korea Racing Authority (KRA) equine hospital with less cooperative inattentive training and reduced performance. Upon admission, the mare showed heightened sensitivity to touch of the hindquarter, reluctance to go forward, frequent urination, and leaning to other competitive horses even during training, standing still, posturing with lowering pelvis and urination, frequent clitoral winking, tail swinging, tail lifting, and training difficulties (Fig. 1). According to horse handlers and the trainer, the mare had expressed these undesirable behaviors with various intensities for more than 1 month. The vital signs (heart rate, respiratory rate, and body temperature) were within the normal ranges. The mare was regarded as normal following physical and lameness examinations by two veterinarians. Complete blood cell count (CBC) results were within normal ranges.

Ultrasound examination and rectal palpation of the reproductive organs were performed and were deemed as being normal and in estrus. The uterus was edematous and presented cervix flowering, and the dominant ovary exhibited a round hypoechoic follicle $(28.1 \mathrm{~mm})$ on ultrasound examination (Fig. 2). Furthermore, estrous behaviors were scored by two veterinarians according to the scoring system for estrus behavioral scores (1-8) proposed by Górecka et al. (3). The estrus behavior score was 8 out of 8 at the first visit. Until ovulation, estrus behavior scores were consistently 8 , and the size of a dominant ovarian follicle on ultrasound examination increased up to $45 \mathrm{~mm}$. The period from the first examination to ovulation was 13 days. The mare had expressed estrous behavior consistent with the development of ovarian follicles throughout the examination period. Therefore, the mare was regarded as requiring estrous suppression.

On the ovulation day, an autoclaved 35-mm glass marble (Mare-bles, Glass Bay studio, OR, USA) was inserted into the uterus (Fig. 3). The perineal region was prepared using a $1 \%$ chlorhexidine solution. A small amount of $0.5 \%$ chlorhexidine cream (Arlico chlorhexidine cream, Arlico, Jincheon, Korea)

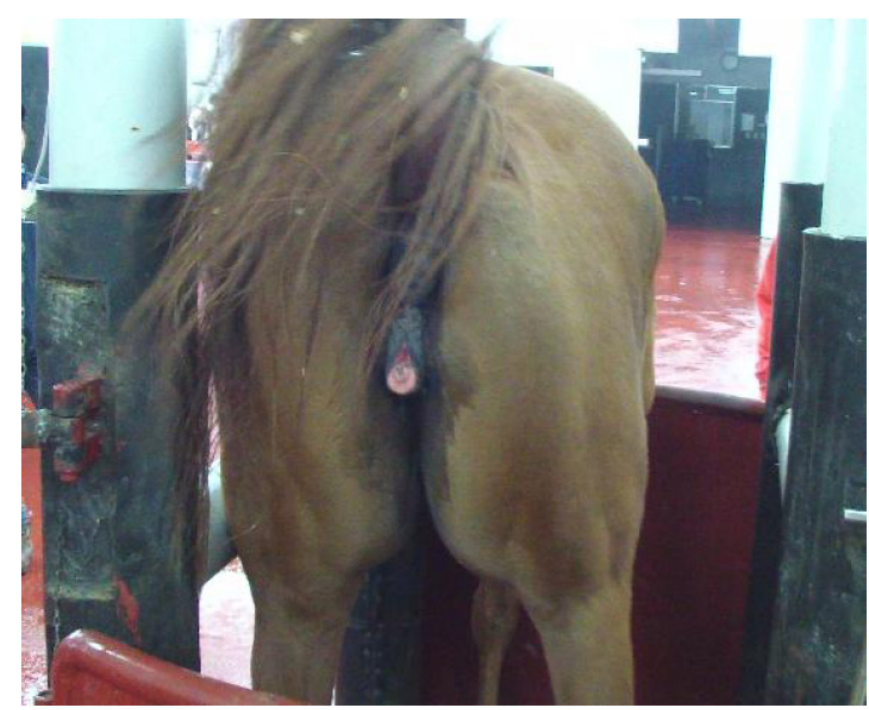

Fig. 1. Tail lifting with clitoral winking in the Thoroughbred mare. 


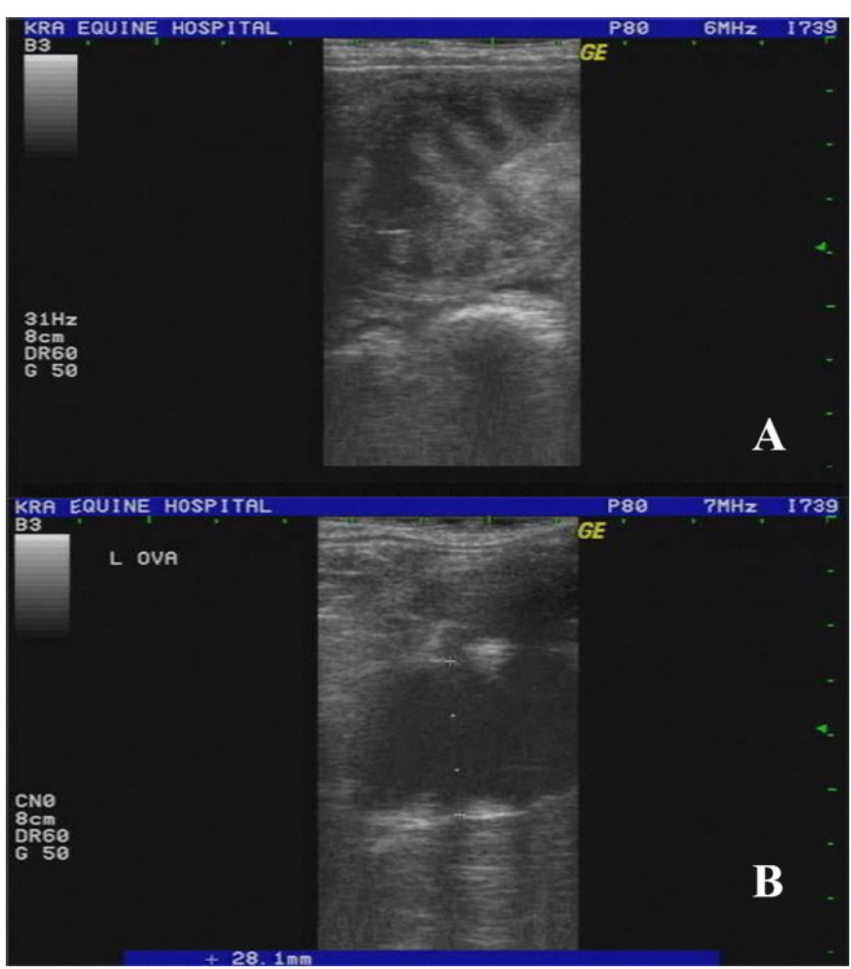

Fig. 2. Cervix flowering of the edematous uterus $(A)$ and $28 \mathrm{~mm}$ of ovarian follicle (B) in the Thoroughbred mare in estrus phase.

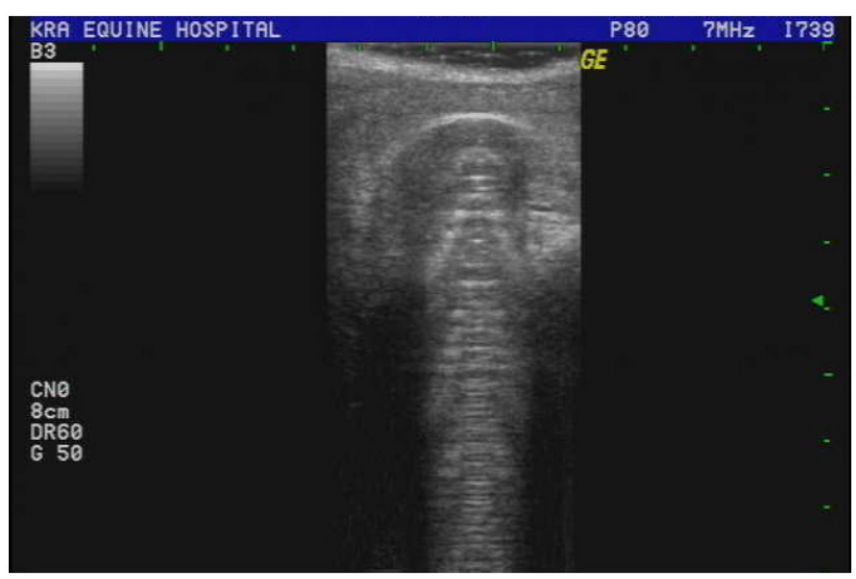

Fig. 3. The ultrasound appearance of intrauterine glass ball at the right uterine horn-body junction.

was applied to the sterilized rectal sleeve (KRUTEX Super Sensitive Gloves with Shoulder Protector, KRUUSE, Langeskov, Denmark), and the marble was inserted into the uterus via the vagina to the cervix and placed in the uterine body. To prevent uterine sepsis, a prophylactic antimicrobial, penicillin G procaine $\left(20,000 \mathrm{IU} / \mathrm{kg}\right.$, IM, PPS ${ }^{\circledR}$, Daesung Microbiological Labs, Euiwang, Korea), was administered after treatment.

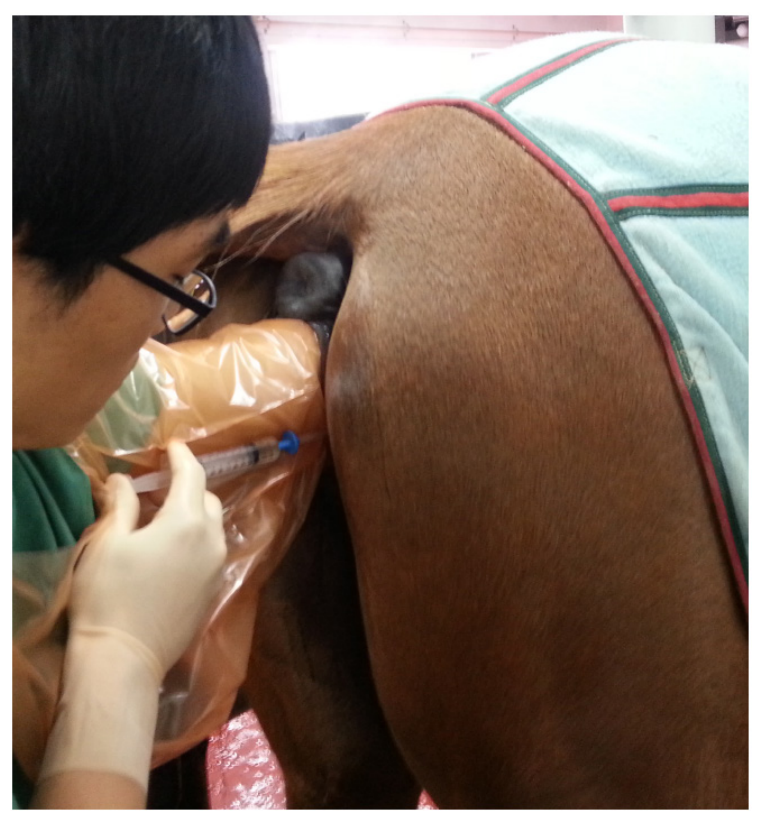

Fig. 4. Insemination catheter was used to administer $1 \mathrm{~mL}$ of peanut oil into the uterus of the Thoroughbred mare.

After 10 days of marble insertion, the mare was brought to the hospital. According to her trainer and horse handlers, the mare expressed similar estrous behaviors. The mare still exhibited clitoral winking, tail lifting, and standing still. The mare's estrus behaviors were scored 6 out of 8 at this time, after which $1 \mathrm{~mL}$ of peanut oil (peanut oil, P2144, Sigma-Aldrich, Saint Louis, MO, USA) was administered into the uterus. In brief, a hand with a sterilized sleeve grabbed the caudal os of the cervix through the vagina and passed a peanut oil-filled insemination catheter (EQUIVET Insemination catheter, KRUUSE, Langeskov, Denmark) through the cervix into the lumen of the uterus (Fig. 4). Instantly, the uterus was kneaded smoothly per rectum for 40 seconds to scatter the oil over the uterine lumen. The undesirable estrous behaviors reduced (score 4 out of 8 ) and the mare returned to training after 2 days of intrauterine oil administration. Since the second treatment, the mare exhibited greater attention in training, and the trainer was satisfied. During 1 month from the second treatment, estrous behavior problems resolved, and no adverse effects were observed.

\section{Discussion}

In the estrus phase of mares, the changes in behavior and reproductive organs are influenced by variations in two hormones, estrogen, and progesterone (15). Elevated estrogen and lower progesterone levels stimulate the central nervous 
system, resulting in the expression of estrous behavior (2). In some mares, temperament, tractability, and trainability are changed by the estrus cycle, resulting in degraded performance (13). There are variations in estrous behavior and lasting periods (13); although the mare displays typical estrous behaviors, it is important to differentiate estrous behaviors related to estrus from other undesirable behaviors irrelevant to estrus to prevent unnecessary treatment initiation (18). According to the trainer, primarily clitoral winking and standing still with raised tails have been continuously observed from late winter. These behaviors are displayed in almost all mares during estrus and a noticeable indication of estrus (18). Therefore, any other potential diseases affecting the behaviors need to be ruled out, and estrous behavior should be related to estrus $(15,18)$. Physical examination, lameness examination, and $C B C$ test revealed that the mare had no abnormal signs. Two veterinarians monitored the signs of estrous behavior using a scoring system for estrus behavioral scores by Górecka et al. (3). Additional ultrasound examination and rectal palpation revealed the estrus stage of the mare, and she was confirmed to express estrus behavior. Taken together, the mare was concluded to require an estrous behavior suppression.

Several treatments have been reported to suppress equine estrous behavior $(8,11,13-15,20)$. The concepts of the treatments include hormonal, surgical, immunologic, and other methods. Meanwhile, there is limited information on their use, efficacy, side effects, and safety (15). Therefore, veterinarians should consider these facts when choosing the method of estrus suppression (18). A highly effective and easy hormonal method is synthetic progesterone administration, using altrenogest $(0.044 \mathrm{mg} / \mathrm{kg})$, once daily per oral, which is the most widely used and recommended agent $(11,15,18)$. Altrenogest can be used for long periods without adverse effects on future reproductive fertility (11). However, the administration of most substances to racing horses in Korea is prohibited and controlled under the rules of racing by the KRA (10). Although altrenogest may be an effective method to suppress estrous behavior, there is an obstacle to applying hormones to athletic horses due to racing rules. In Korea, almost all drug administration should be withdrawn at least 10 days before participating in a race, during which time the horse may display estrus signs. The withdrawal period of altrenogest for racing horses is 14 days in Korea (10). Mares return to estrus within 4-5 days after discontinuation of the altrenogest treatment and continue to cycle normally (17). Therefore, the administration of altrenogest is not applicable to suppress estrous behavior among racing horses in Korea. Furthermore, other hormonal treatments have limitations in their application, including limited effects, side effects, lack of long-term safety information, and drug unavailability in Korea. Ovariectomy can be considered for suppressing estrus in mares as a last resort $(11,15)$. However, it is a non-reversible and expensive procedure, resulting in the loss of possibility for further reproduction $(11,15,18)$. Moreover, there are some risks related to the surgical procedure and $35 \%$ of continued estrous behavior postoperatively (5). While a high level of estrogen usually stimulates estrous behavior in other species, estrous behavior in mares can be expressed in the absence of progesterone, despite the lack of estrogen (15). Gonadotrophin-releasing hormone (GnRH) vaccination blocks the pituitary to release gonadotropins, leading to a suspension of estrus cyclicity $(15,20)$. This method suspends ovarian activity and suppresses estrus for 25-30 weeks (20). Ninety-two percent of vaccinated mares resume the ovarian cycle in two years (20). Although $\mathrm{GnRH}$ vaccination appears to be a simple, long-term effective, safe, and reversible treatment to suppress estrus in mares, it is not approved for use in mares in most countries, except Australia, and is currently unavailable in Korea $(15,20)$.

It was decided to initiate the therapy without drugs regarding efficacy, safety, side effects, reversibility, drug availability, and tight racing schedule to be continuous participation in 2-4 weeks intervals. Due to the KRA regulation of the drug withdrawal period, intrauterine glass marble and peanut oil were chosen for racing horses. At ovulation, a $35-\mathrm{mm}$ diameter glass ball, which is the optimal size for mares (14), was inserted into the uterus. Intrauterine glass ball seems to mimic the conceptus and inhibit prostaglandin release from the endometrium, in turn preventing luteolysis and maintaining the corpus luteum $(14,19)$. Estrus suppression occurs by prolonged elevation in progesterone production by persisting luteal function $(14,15)$. The effects of cyclicity prevention and extended luteal period are reported to be $39 \%$ and 87 days, respectively (14). Although the heightened sensitivity to touch of the hindquarter and frequent urination subsided, the mare still displayed clitoral winking, standing still, and tail lifting with reduced intensity due to the relatively low efficacy of glass ball and individual variation of the mare. To further suppress estrous behavior, $1 \mathrm{~mL}$ of peanut oil was inserted post-ovulation day 10 as an additional treatment. In 2011, Wilsher and Allen reported estrus suppression of $92 \%$ in 12 mares for approximately 3 months (22). Peanut oil contains $14-43 \%$ of linoleic acid that appears to prevent prostaglandin F2 $\alpha$ synthesis through mechanisms, such as reduced availability of its precursor arachidonic acid, and increased competition with arachidonic acid for binding cyclooxygenase (COX) or inhibition of COX synthesis and activity, although 
the precise mechanism is not fully understood (22). In this case, the mare displayed no estrous behavior after peanut oil treatment for over 30 days until loss to follow-up due to retirement by injury. Although it was not traced the effect further due to a limb injury occurring during training, the trainer, horse handlers, and the jockey were satisfied with the mare while training and reached a consensus on the estrous behavior suppression after treatment.

Considering the efficacy, commercial availability, safety, side effects, and withdrawal period, there are almost no effective single-dose medications or treatment currently available for suppressing the estrous behavior of mares for a prolonged period in Korea. Meanwhile, peanut oil treatment is an effective, safe, simple, inexpensive, and available choice for athletic horses suffering from estrus discomfort that are acceptable per the rules of racing in Korea. Therefore, intrauterine peanut oil deposition is a suitable choice to control estrous behavior for a prolonged period in athletic horses in Korea.

\section{Conflicts of Interest}

The authors have no conflicting interests.

\section{References}

1. British Horseracing Authority Web site. Rules of racing and guides. Available at: https://www.britishhorseracing.com/regulation/ rules-guides/. Accessed Jun 1, 2021.

2. Davies-Morel M. Control of reproduction. In: Davies-Morel M, editor. Breeding horses. Oxford: Blackwell Publishing. 2005: 37-56.

3. Górecka A, Jezierski TA, Słoniewski K. Relationships between sexual behaviour, dominant follicle area, uterus ultrasonic image and pregnancy rate in mares of two breeds differing in reproductive efficiency. Anim Reprod Sci 2005; 87: 283-293.

4. Hedberg Y, Dalin AM, Forsberg M, Lundeheim N, Hoffmann B, Ludwig C, et al. Effect of ACTH (tetracosactide) on steroid hormone levels in the mare. Part A: effect in intact normal mares and mares with possible estrous related behavioral abnormalities. Anim Reprod Sci 2007; 100: 73-91.

5. Hooper RN, Taylor TS, Varner DD, Blanchard TL. Effects of bilateral ovariectomy via colpotomy in mares: 23 cases (1984-1990). J Am Vet Med Assoc 1993; 203: 1043-1046.

6. Irish Horseracing Regulatory Board Web site. Rules of racing and Irish national hunt steeplechase rules. Available at: https://www. ihrb.ie/pdf.php?f=RuleBook.pdf. Accessed Jun 1, 2021.

7. Japan Racing Association Web site. Rules of racing. Available at: https://japanracing.jp/en/horsemen/jra_rules/rules_racing.html. Accessed Jun 1, 2021.

8. Jorgensen JS, Mansmann RA. Estrous cycle and performance in athletic mares. In: Ross MW, Dyson SJ, editors. Diagnosis and management of lameness in the horse. Saint Louis: W.B. Saunders. 2003: 480-482.

9. Jorgensen JS, Vivrette S, Correa M, Mansmann RA. Significance of the estrous cycle on athletic performance in mares. In: Proceedings of the 42nd Annual Meeting of the American Association of Equine Practitioners. Denver: American Association of Equine Practitioners, 1996: 98-100.

10. Korea Racing Authority Web site. KRA rules of racing. Available at: https://race.kra.co.kr/pdf/rulesofracing.pdf. Accessed Jun 1, 2021.

11. McCue PM. Estrus suppression in performance horses. J Equine Vet Sci 2003; 23: 342-344.

12. McGreevy P. Behavior of the mare. In: McGreevy P, editor. Equine behavior. Oxford: W.B. Saunders. 2004: 265-290. https://doi. org/10.1016/B978-0-7020-2634-8.50017-4.

13. McDonnell SM. Estrus cycle-related performance problems. J Equine Vet Sci 1997; 17: 196.

14. Nie GJ, Johnson KE, Braden TD, Wenzel JGW. Use of an intra-uterine glass ball protocol to extend luteal function in mares. J Equine Vet Sci 2003; 23: 266-273.

15. Nie GJ. Estrus suppression. In: Samper J, McKinnon A, editors. Current therapy in equine reproduction. Saint Louis: W.B. Saunders. 2007: 26-31.

16. Norwegian Trotting Association Web site. Competition rules for Norwegian trotting 2019. Available at: http://www.travsport.no/ Global/Logoer/Norwegian\%20racing\%20regulations.pdf. Accessed Jun 1, 2021

17. Papich MG. Altrenogest. In: Papich handbook of veterinary drugs. 5th ed. St. Louis: W.B. Saunders. 2021: 25-26.

18. Pryor P, Tibary A. Management of estrus in the performance mare. Clin Tech Equine Pract 2005; 4: 197-209.

19. Rivera Del Alamo MM, Reilas T, Kindahl H, Katila T. Mechanisms behind intrauterine device-induced luteal persistence in mares. Anim Reprod Sci 2008; 107: 94-106.

20. Schulman ML, Botha $A E$, Muenscher SB, Annandale $C H$, Guthrie AJ, Bertschinger HJ. Reversibility of the effects of GnRH-vaccination used to suppress reproductive function in mares. Equine Vet J 2013; 45: 111-113.

21. Vanderwall D. Prolonging function of the corpus luteum to suppress estrus in mares. In: Proceedings of the 59th Annual Convention of the American Association of Equine Practitioners. Nashiville: American Association of Equine Practitioners, 2013: 342-349.

22. Wilsher S, Allen WR. Intrauterine administration of plant oils inhibits luteolysis in the mare. Equine Vet J 2011; 43: 99-105. 